\title{
Regulation of Blos1 by IRE1 prevents the accumulation of Huntingtin protein aggregates
}

Donghwi Bae, Rachel Elizabeth Jones, and Julie Hollien*

School of Biological Sciences and the Center for Cell and Genome Science, University of Utah. Salt Lake City, UT.

*Correspondence: juliehollien@gmail.com

\begin{abstract}
Huntington's Disease is characterized by accumulation of the aggregation-prone mutant Huntingtin (mHTT) protein. Here, we show that expression of mHTT in mouse cultured cells activates IRE1, the transmembrane sensor of stress in the endoplasmic reticulum, leading to degradation of the Blos $1 \mathrm{mRNA}$ and repositioning of lysosomes and late endosomes toward the microtubule organizing center. Overriding Blos 1 degradation results in accumulation of larger mHTT aggregates and increased cell death. Although mHTT is degraded by macroautophagy when highly expressed, we show that prior to the formation of large aggregates, mHTT is degraded via an ESCRT-dependent, endosomal microautophagy pathway. This pathway is enhanced by Blos 1 degradation and appears to protect cells from a toxic, less aggregated form of mHTT.
\end{abstract}

\section{Condensed title}

Blos 1 regulation protects from Huntingtin protein aggregation 


\section{Introduction}

Protein aggregation underlies several common and devasting neurodegenerative diseases, for which there are no cures ${ }^{1,2}$. However, the distinctive protein deposits that form within the brain are not always correlated with the specific neurons whose death or loss of function leads to the symptoms experienced by patients ${ }^{3-5}$. This observation has led to the idea that smaller, oligomeric forms of aggregation-prone proteins are more likely to be toxic, by interfering with normal cellular processes $^{6}$. In Huntington's disease, for example, large protein aggregates of the mutant Huntingtin protein (mHTT) are thought to be somewhat protective ${ }^{7,8}$. These aggregates can be degraded by macroautophagy (MA), which encloses aggregates in double-membraned autophagosomes that then fuse with lysosomes for degradation ${ }^{9,10}$. Whether smaller, potentially more toxic, oligomers are also degraded by MA or by distinct pathways is not clear.

Aggregating proteins tend to accumulate at the juxtanuclear microtubule organizing center (MTOC), as a result of retrograde (minus end-directed) trafficking on microtubules, a process that is dependent on the ubiquitin-binding adapter protein HDAC6 ${ }^{10,11}$. Disruption in trafficking hinders both the coalescence into larger aggregates and the degradation of these proteins ${ }^{11}$. Retrograde trafficking of lysosomes has been observed in cellular models of Huntington's and Parkinson's diseases ${ }^{12,13}$, although long-distance trafficking along axons is often disrupted in these diseases ${ }^{14}$.

We have found that retrograde trafficking and clustering of lysosomes and late endosomes (LEs) near the MTOC can result from a specific pathway induced by stress in the endoplasmic reticulum $(\mathrm{ER})^{15}$. Inositol requiring enzyme 1 (IRE1), a conserved sensor of ER stress and key mediator of the unfolded protein response, is a nuclease that cleaves the mRNA encoding the transcription factor XBP1 to initiate its splicing and activation, leading to the upregulation of many 
genes involved in ER homeostasis. IRE1 also cleaves several other mRNAs, initiating their degradation through the Regulated IRE1-Dependent Decay (RIDD) pathway ${ }^{16,17}$. One of the key targets of RIDD is the mRNA encoding biogenesis of lysosome-related organelles complex 1 subunit 1 (BLOC1S1, or BLOS1). BLOS1 is a member of the BLOC1-related complex (BORC), which links lysosomes to kinesin, allowing for their trafficking toward the periphery of the cell ${ }^{18}$. Degradation of the Blos 1 mRNA by RIDD during ER stress leads to the repositioning of LE/ lysosomes to the MTOC and to the enhanced degradation of ubiquitinated proteins ${ }^{15}$.

Because neurodegenerative diseases such as Huntington's Disease often display markers of ER stress ${ }^{19,20}$, we hypothesized that the degradation of Blos 1 may contribute to clearance of disease-associated aggregating proteins by trafficking degradative organelles to the cell center. Here we show that expression of the aggregation-prone Huntingtin protein induces degradation of Blos 1 mRNA, which protects cells from apoptosis and prevents the accumulation of aggregates by enhancing an alternative pathway to degradation.

\section{Results \& Discussion}

To test whether expression of the mutant Huntingtin protein induces IRE1 and the RIDD pathway, we constructed stable MC3T3-E1 cell lines overexpressing exon 1 of either the wildtype Huntingtin protein (wtHTT), which contains a string of 23 Gln residues, or a disease-causing mutant Huntingtin (mHTT), which contains 145 Gln's, each tagged with GFP and under the control of a doxycycline-inducible promotor (Fig 1A). We transfected these HTT plasmids into cell lines expressing either Rfp (as a control) or a stabilized version of the Blos 1 mRNA (Blos $1^{s}$ ), which contains a silent point mutation rendering it resistant to degradation by IDD $^{17}$. We 
previously showed that these Blos $1^{s}$-expressing cells override the repositioning of LE/lysosomes during ER stress ${ }^{15}$. Addition of doxycycline $(4.5 \mu \mathrm{M}, 72 \mathrm{~h})$ to control cells and the subsequent expression of mHTT, but not wtHTT, led to the activation of IRE1 as assessed by Xbpl splicing (Fig 1B), degradation of RIDD targets (Fig 1C-D), and repositioning of LE/lysosomes to the MTOC (Fig 1E, F). As expected, when we induced mHTT expression in cells expressing $B l o s 1^{s}$, IRE1 was activated but LE/lysosomes did not reposition (Fig 1B-F).

Large aggregates of mHTT were visible in only about $13 \%$ of control cells in these experiments, suggesting that extensive aggregation is not required to induce IRE1 (Fig 2A). Furthermore, when we used more mild induction conditions $(0.9 \mu \mathrm{M}$ doxycycline, $36 \mathrm{~h})$ such that the mHTT-GFP fluorescence remained diffuse, cells still degraded Blos 1 mRNA and repositioned LE/lysosomes (Fig 1G-H and 2A). This is consistent with previous studies indicating that mHTT activates ER stress pathways by interfering with the retrotranslocation and proteasomal degradation of misfolded proteins from the $\mathrm{ER}^{21,22}$, rather than through a mechanism dependent on extensive aggregation.

The lack of visible aggregates in most of our control cells suggested that MC3T3-E1 cells effectively degrade mHTT or prevent its aggregation. The aggregates that did appear were characteristically juxtanuclear and surrounded by lysosomes (Fig 2C), suggesting that lysosome positioning may contribute to mHTT degradation. Accordingly, over 50\% of cells co-expressing $B l o s 1^{s}$ contained juxtanuclear mHTT aggregates, which were larger than in control cells and were not surrounded by lysosomes (Fig. 2A-C). To determine the extent of mHTT accumulation in these cells, we measured the fluorescence of its GFP tag by flow cytometry. Blos $1^{s}$-expressing cells had a median mHTT-GFP signal over 25 times higher than control cells (Fig 2D), although the mRNA levels were similar between the two cell lines (Fig 2E). These data suggest that RIDD of Blos1 is 
important for degrading mHTT and avoiding the accumulation of large aggregates.

Mutant HTT has previously been shown to be degraded by macroautophagy (MA). Consistently, treatment of cells with high levels of doxycycline $(4.5 \mu \mathrm{M})$ led to higher levels of lipidated LC3B (LC3B-II, Fig. 3A-B), a marker for the induction of MA. However, at lower concentrations of doxycycline $(0.9 \mu \mathrm{M})$, LC3B-II levels remained low and indistinguishable from cells without doxycycline. It was not possible to determine precisely whether the increase in LC3B-II levels in high doxycycline was due to increased induction of MA vs. less efficient degradation of LC3B-II following the fusion of autophagosomes with lysosomes, because inhibiting lysosomal function for the full timescale of the experiment led to extensive cell death. However, including chloroquine, which blocks the acidification of lysosomes, for the final 2 hours did lead to increased LC3B-II in all conditions (Fig. 3A-B). To test the idea that MA is responsible for degradation of mHTT only at high expression levels, we used RNAi to deplete the mRNA encoding the essential MA factor ATG7, induced mHTT expression with either 0.9 or $4.5 \mu \mathrm{M}$ doxycycline, and measured the accumulation of mHTT-GFP by flow cytometry. In the high doxycycline conditions, Atg7 knockdown led to a large increase in mHTT levels, whereas in the low doxycycline conditions, Atg7 knockdown had no effect (Fig 3C). In contrast, knockdown of either Hdac6 (which is important for trafficking aggregating proteins to the MTOC) or Vps22 (which is important for degradation of ubiquitinated aggregates during ER stress ${ }^{15}$ ), led to accumulation of mHTT in both doxycycline concentrations (Fig 3C).

These results suggest that mHTT induces MA and is degraded by MA only when expressed at high levels, potentially when larger aggregates begin to form. To further test this idea, we measured both aggregate formation (by microscopy) and MA induction (by immunoblot of LC3BII) over time in cells expressing Blos $1^{s}$, which accumulate aggregates when mHTT expression is 
induced with either low or high concentrations of doxycycline. The induction of MA coincided with the appearance of large, visible aggregates, after 24 hours of $4.5 \mu \mathrm{M}$ doxycycline or 36 hours of $0.9 \mu \mathrm{M}$ doxycycline (Fig 3D-F). We therefore propose that while MA is involved in mHTT degradation, a distinct pathway is necessary for its degradation at lower expression levels and/or smaller aggregation states, before large aggregates form. Furthermore, because Blos $1^{s}$ affects mHTT degradation in both cases, RIDD of Blos 1 appears to enhance this MA-independent pathway.

To explore the mechanism of mHTT degradation in the low expression and subaggregation state, we depleted cells of various factors by RNAi, induced mHTT expression with $0.9 \mu \mathrm{M}$ doxycycline, and measured its accumulation by flow cytometry. Similar to the depletion of Atg7, depletion of Lamp2 (which is important for chaperone-mediated autophagy) did not affect mHTT levels (Fig 3G). However, we observed strong mHTT accumulation in cells depleted of factors involved in the endosomal sorting complex required for transport (ESCRT) pathway, which is responsible for the inward budding of vesicles into LE's or multivesicular bodies ${ }^{23}$. The ESCRTs are composed of distinct complexes (ESCRTs -0, -I, -II, -III, and VPS4) that sort cargo and deform the limiting membrane of the endosome to allow for the formation of LEs, which then fuse with lysosomes for cargo degradation. In our experiments, mHTT accumulated following knockdown of the ESCRT-I factor Tsg101, the ESCRT-II factor Vps22, the ESCRT-III accessory factor Alix, and $\operatorname{Vps} 4 A$, which is essential for the disassembly and recycling of ESCRT machinery (Fig 3C,G).

ESCRT-0 also appears to be critical for preventing the accumulation of mHTT. ESCRT-0 is a heterotetromer of two HRS and two STAM1 or STAM2 subunits, and it binds both endosomal membranes and ubiquitinated targets and initiates the multivesicular body pathway ${ }^{23}$. Depletion of Stam1 or 2 led to dramatic accumulation of mHTT (Fig 3G), whereas depletion of the Hrs1 led to 
cell death upon induction of mHTT expression (Fig 3H). Furthermore, depletion of Rab35, which encodes a small GTPase that regulates $\mathrm{HRS}^{24,25}$, also led to strong mHTT accumulation (Fig. $3 G)$.

Based on these data, we conclude that several core components of the ESCRT machinery are important for the degradation of mHTT. ESCRTs are involved in many cellular mechanisms, and directly or indirectly affect all three major autophagic pathways ${ }^{26}$, i.e. MA, chaperonemediated autophagy, and endosomal macroautophagy (eMI). However, the lack of dependence on MA or chaperone-mediated autophagy for mHTT degradation at low induction levels suggests that the ESCRTs contribute to mHTT degradation directly via $\mathrm{eMI}^{27}$. Although the mechanistic details are not yet well-understood for mammalian cells, eMI is characterized by the delivery of cytosolic cargo, such as glycolytic proteins, to LEs in a manner that relies on several ESCRT proteins, including TSG101 and VPS4 ${ }^{26,28}$.

We next carried out apoptosis assays to test the relative toxicity of mHTT in both control and $B l o s 1^{s}$-expressing cells after treatment with low or high doxycycline concentrations. Control cells were much more resistant to apoptosis, indicating that degrading Blos 1 mRNA not only prevents the accumulation of mHTT aggregates but also protects cells from death. Surprisingly,

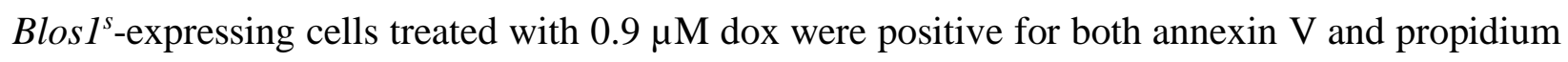
iodide, indicative of late apoptosis, whereas cells treated with $4.5 \mu \mathrm{M}$ dox were more likely to be stained for annexin $\mathrm{V}$ only, indicative of early apoptosis (Fig. 3I). These differences cannot be explained by the amount of mHTT or the number of aggregates at the time of the assay ( $36 \mathrm{~h}$ after doxycycline addition), as these were indistinguishable between the two doxycycline treatments (Fig 2D and 3D). However, due to the delay in forming large aggregates in the lower expression regime (Fig 3D), we suggest that these cells are exposed to a more toxic, sub-aggregation form of 
mHTT for a longer time compared to the high expression regime where mHTT accumulates in large aggregates more rapidly.

To test the generality of these findings and the ability of Blos 1 regulation to enhance mHTT degradation in a neuronal cell line, we transfected wt or mHTT-GFP into mouse Neuro2A (N2A) cells, with or without Blos $1^{s}$. As in the MC3T3-E1 cells, expression of mHTT led to the degradation of the Blosl mRNA, and co-expression of Blos $1^{s}$ led to increased accumulation of mHTT but not wtHTT (Fig 4A-B). We then asked whether mHTT is degraded by eMI and/or MA in these cells. In control cells not expressing Blos $1^{s}$, knockdown of the ESCRT-0 factor Staml, but not the MA factor Atg7, led to increased mHTT accumulation (Fig 4C). There was no difference between mHTT levels in control vs. Blos $1^{s}$ cells after depletion of Stam1. In contrast, Atg7 knockdown led to increased mHTT accumulation beyond the effects of $B l o s I^{s}$ expression. Taken together, these data suggest that the effect of Blosl regulation is primarily on eMI. When eMI is crippled by Blos $1^{s}$ expression, knockdown of Staml has no further effect, whereas knockdown of Atg7 (which normally does not affect mHTT degradation in these cells) exacerbates Blos $1^{s}$ expression by preventing mHTT degradation by MA.

Unlike in MC3T3-E1 cells, LAMP1 structures in the N2A cells were predominantly juxtanuclear even in untransfected cells or cells transfected with wtHTT, and this localization was unaffected by Blos $1^{s}$ expression (Fig. 4D). RAB7 structures, in contrast, were peripherally distributed in a higher fraction of cells, and did appear to be more juxtanuclear upon expression of mHTT but not wtHTT (Fig 4E). Blos $1^{s}$ expression prevented this repositioning, similar to our results in MC3T3-E1 cells. Because LAMP1 stains both lysosomes and LEs, whereas RAB7 is typically more specific to LEs, we suggest that the repositioning of LEs may account for the effects of Blos 1 degradation, at least in the case where lysosomes are already available near the MTOC. 
This is consistent with a primary effect of Blosl on eMI. Alternatively, as suggested by the large fraction $(>50 \%)$ of untransfected cells displaying juxtanuclear RAB7 structures, Blos 1 regulation may affect eMI in ways unrelated to the simple trafficking and localization of these organelles. In support of this idea, BORC, the BLOS1-containing complex responsible for LE/lysosome trafficking, has also been shown to regulate autophagosome-lysosome fusion ${ }^{29}$ and LE/lysosome $\operatorname{size}^{30}$; and BLOS1 itself also participates in a variety of related cellular functions both through its role in the biogenesis of lysosome related organelles complex (BLOC1) and potentially independently ${ }^{31,32}$.

Our overall model is that oligomers or small aggregates of mHTT are degraded by eMI, in a manner that depends on the degradation of Blosl by the RIDD pathway, potentially due to the repositioning of LEs to the MTOC (Fig. 4F). When this pathway for degradation is compromised (as in cells expressing $B l o s 1^{s}$ ), mHTT accumulates in juxtanuclear aggregates and MA is induced. Although the regulation of Blos 1 has not been explored in neurodegenerative diseases, the tau protein, which is involved in the pathogenesis of Alzheimer's disease, has also been shown to be degraded by both eMI and MA ${ }^{25,33}$. More generally, dysfunction of the ESCRT machinery, including ESCRT-0, has been linked to neurodegeneration and the accumulation of intracellular protein aggregates $^{34,35}$.

Why are different degradation pathways used for the clearance of mHTT? It is likely that MA is necessary for the removal of aggregates once they reach a certain size, but why cells would use eMI rather than MA in the case of smaller oligomers is an open question. We speculate that by enclosing these smaller aggregates within LEs, neurons retain the option of not only degrading these toxic species within their own lysosomes, but also secreting them outside the cell within exosomes, upon fusion of the LEs with the plasma membrane. This mechanism, which is emerging 
as a common feature of neurodegenerative diseases, may help to protect neurons from toxicity when lysosomal capacity is not sufficient, while also potentially explaining the propagation of aggregates to target cells that take up these vesicles ${ }^{36,37}$.

\section{Acknowledgments}

We thank M. Babst and members of the Hollien lab for discussions. This research was supported by NIH R35 GM119540. The authors declare no competing financial interests.

\section{Author contributions}

Conceptualization, investigation, review \& editing: all authors. Original draft: DB and JH.

\section{Figure Legends}

\section{Figure 1. Expression of mHTT but not wtHTT induces IRE1.}

(A) We constructed stable MC3T3-E1 cells expressing either Rfp (labelled as control, grey bars throughout), or Blosls (blue bars) and either wt or mHTT-GFP under a doxycycline-inducible promoter. (B) We induced expression using doxycycline (dox; $4.5 \mu \mathrm{M}, 72 \mathrm{~h}$ ), collected RNA, and measured Xbpl splicing by PCR followed by gel analysis. (C-D) Using samples from A, we measured the relative mRNA abundance of the RIDD targets Hgsnat and Blosl by real-time quantitative PCR (qPCR). (E-F) We induced expression of mHTT-GFP (4.5 $\mu \mathrm{M}$ dox, $72 \mathrm{~h})$, then fixed and stained cells for LAMP1 to image LE/lysosomes (scale bar, $10 \mu \mathrm{m}$ ). F shows the quantification of 3 independent experiments (100-120 cells per treatment per experiment). (G) We induced expression of wt or mHTT-GFP $(0.9$ or $4.5 \mu \mathrm{M}$ dox, $36 \mathrm{~h})$, and measured relative Blos1 
abundance by qPCR. (H) We induced expression of mHTT as in G and quantified LE/lysosome localization as in E-F. For all graphs: independent experiments are indicated by symbol type, and bars show the average of 3 experiments. ${ }^{*}, \mathrm{p}<0.05$, paired t-test followed by Holm-Bonferroni correction for multiple comparisons.

Figure 2. Overriding Blos1 degradation leads to the accumulation of mHTT aggregates.

(A-B) We induced expression (0.9 or $4.5 \mu \mathrm{M}$ dox, $36 \mathrm{~h}$ ) of mHTT-GFP in cells expressing $R f p$ (control, grey bars) or Blos $1^{s}$ (blue bars). We then imaged live cells and quantified the fraction of cells with a prominent juxtanuclear aggregate (A) and measured the median diameter of the aggregate using ImageJ (B). (C) Representative images of control and Blos $1^{s}$-expressing cells, fixed and stained for LAMP1. mHTT-GFP is the residual GFP fluorescence following fixation. (D) We induced expression of mHTT-GFP as in A,B and measured the median abundance of mHTT-GFP by flow cytometry. (E) We induced mHTT-GFP expression as in D, collected RNA and measured relative mHTT-GFP mRNA abundance by qPCR. For all graphs: independent experiments are indicated by symbol type, and bars show the average of 3 experiments. *, $\mathrm{p}<0.05$, paired t-test followed by Holm-Bonferroni correction for multiple comparisons.

Figure 3. The degradation mechanism and toxicity of mHTT depends on induction level.

(A-B) We induced expression of mHTT-GFP in control MC3T3-E1 cells (0.9 or $4.5 \mu \mathrm{M}$ dox, 36 h), with or without chloroquine $(\mathrm{CQ}, 60 \mu \mathrm{M})$ included for the final $2 \mathrm{~h}$. We then measured LC3B processing by immunoblotting. The open triangle indicates increasing amounts of protein were loaded. Quantification of three independent immunoblots are shown in B. (C) We used RNAi to 
deplete MC3T3-E1 cells of the indicated factors, then induced mHTT-GFP expression (36 h) and measured the median fluorescence of cell populations by flow cytometry. ${ }^{*}, \mathrm{p}<0.05$ compared to the control knockdown at the same dox concentration. (D-F) We induced expression of mHTTGFP in MC3T3-E1 cells expressing Blos $1^{s}$ with either $0.9 \mu \mathrm{M}$ (open symbols) or $4.5 \mu \mathrm{M}$ (closed symbols) dox, and then measured the fraction of cells with large GFP aggregates (by microscopy, C) and the extent of MA induction (by LC3B immunoblot, E-F) over time. *, p < 0.05 comparing 0.9 vs $4.5 \mu \mathrm{M}$ dox. (G) We used RNAi to deplete MC3T3-E1 cells of the indicated factors, induced mHTT-GFP with low dox concentrations $(0.9 \mu \mathrm{M}, 36 \mathrm{~h})$, and measured the median fluorescence as in B. For this panel, the data were analyzed by one-way ANOVA followed by Tukey's Honest Significant Difference test (*, $\mathrm{p}<0.05$ compared to the control knockdown). (F) We used RNAi to deplete MC3T3-E1 cells of Hrs1, induced mHTT-GFP with low dox concentrations $(0.9 \mu \mathrm{M}$, $36 \mathrm{~h}$ ), aspirated floating cells from the plate and counted remaining living cells. (I) We induced expression of mHTT-GFP in cells expressing $R f p$ (control, grey bars) or Blos $1^{s}$ (blue bars) for 36 $\mathrm{h}$, then measured the degree of apoptosis using annexin $\mathrm{V}$ and propidium iodide staining followed by flow cytometry. E=early apoptosis (staining with annexin $\mathrm{V}$ only), L=late apoptosis (staining with both annexinV and PPI). For all graphs: independent experiments are indicated by symbol type, and bars show the average of 3 experiments. *, $\mathrm{p}<0.05$, paired t-test followed by HolmBonferroni correction for multiple comparisons, except as noted in panel E.

Figure 4. Blos1 regulation protects cells from mHTT accumulation in N2A cells by enhancing endosomal microautophagy.

(A,B) We cotransfected N2A cells with Rfp (labelled as control, grey bars), or Blos $1^{s}$ (blue bars) and either wt or mHTT-GFP under a constitutive promoter. After $48 \mathrm{~h}$, we measured Blos1 mRNA 
levels by qPCR (A) and the accumulation of HTT-GFP by flow cytometry (B). (C) We used RNAi to deplete N2A cells of Stam1 or Atg7, then transfected and measured the accumulation of HTTGFP as in B. (D,E) We compared untransfected N2A cells to those transfected as in A-B. We stained cells with antibodies for LAMP1 (D) or RAB7 (E) and scored the fraction of cells with juxtanuclear foci. (F) Model for the degradation of small mHTT aggregates. For all graphs: independent experiments are indicated by symbol type, and bars show the average of 3 experiments. ${ }^{*}, \mathrm{p}<0.05$, paired t-test followed by Holm-Bonferroni correction for multiple comparisons.

\section{Materials and Methods}

\section{Cell Culture, plasmids, and transfections}

We cultured MC3T3-E1 cells in MEM $\alpha$ with nucleosides, L-glutamine, and no ascorbic acid (Life Technologies) supplemented with $10 \% \mathrm{FBS}$ at $37^{\circ} \mathrm{C}$ and $5 \% \mathrm{CO} 2$. We cultured N2A cells in DMEM supplemented with 10\% FBS. Both cell lines were purchased from American Type Culture Collection (ATCC).

We subcloned PCR products encoding exon 1 of either wild-type (23 CAG repeats) or mutant (145 CAG repeats) Huntingtin (HD Community Biorepository, ref\# CHDI-90000038 for wild-type Huntingtin and CHDI-90000040 for mutant huntingtin), followed by GFP, downstream of the human EF1 $\alpha$ or the doxycycline-inducible tight TRE promotor. The Blos $1^{s}$ construct described previously ${ }^{15}$ contains a silent point mutation (G360C) in the coding sequence that prevents its degradation by $\operatorname{RIDD}^{17}$.

To generate the MC3T3-E1 cell lines used in this study, we used control cells (expressing 
Rfp) and Blos $1^{s}$-3xFlag-expressing cells described previously ${ }^{15}$, and carried out additional transfections using Lipofectamine 2000 (Invitrogen). Into each cell line, we transfected a TetR plasmid (addgene: pMA2640 38 ), which expresses the reverse tetracycline-regulated transactivator to allow for doxycycline-inducible expression, and selected for stable expression using blasticidin $(5 \mu \mathrm{g} / \mathrm{mL})$. We then transfected the wt or mHTT plasmid and selected using puromycin $(2 \mu \mathrm{g} / \mathrm{mL})$. We maintained cells in these antibiotics until one passage before each experiment.

We transiently transfected N2A cells using Lipofectamine 3000 (Invitrogen). For each experiment we transfected plasmids expressing with wt or mHTT-GFP under the constitutive EF1 $\alpha$ promoter, along with plasmids expressing either $R f p$ (as a control) or Blos $1^{s}-3 x$ Flag. We allowed the cells to recover for 48 hours before assaying.

\section{RNA interference}

For siRNA knockdown experiments in MC3T3-E1 cells, we used RNAiMAX (Invitrogen) to transfect two siRNAs (Sigma-Aldrich) for each target mRNA. As a control we transfected siRNAs designed to not target any mammalian mRNAs (Qiagen). We collected cells 48 hours after transfection, washed in PBS, and measured the GFP signal intensity using flow cytometry as described below.

For RNAi in N2A cells, we first transfected cells with siRNAs as for MC3T3-E1 cells. We allowed cells to recover for 24 hours before transfecting with plasmids containing either wt or mHTT-GFP and Rfp or Blos $1^{s}-3 x F l a g$, all controlled by the EF1 $\alpha$ promoter, with Lipofectamine 3000 (Invitrogen). We then waited an additional 48 hours before measuring GFP signal by flow cytometry. 


\section{Immunostaining and microscopy}

For immunostaining, we grew cells on glass coverslips, fixed using $4 \%$ paraformaldehyde and $1 \mathrm{mM} \mathrm{MgCl}$ in PBS (37 C, $15 \mathrm{~min}$ ), and permeabilized using 0.2\% Triton $\mathrm{X}-100$ and $1 \mathrm{mM}$ $\mathrm{MgCl}$ in PBS (room temperature, $20 \mathrm{~min}$ ). We then incubated cover slips in blocking solution (2\% BSA, $0.02 \%$ Tween-20, and $1 \mathrm{mM} \mathrm{MgCl}$ in PBS, $10 \mathrm{~min}$ ), then with primary antibodies in blocking solution (room temperature, 1 hour). We used primary antibodies for LAMP (DSHB, 1D4B-s, $2 \mu \mathrm{g} / \mathrm{mL}$ ) or RAB7 (Cell Signaling 9367, 1:100). We washed 3 times (0.02\% Tween-20, $1 \mathrm{mM} \mathrm{MgCl}, \mathrm{PBS}$ ), incubated with secondary antibodies (1 hour), and washed again. We then mounted the coverslips on slides in ProLong Diamond Antifade mountant with DAPI (Invitrogen). For live cell imaging of wt and mHTT-GFP, we plated cells on glass-bottom dishes.

To image cells, we used an Olympus IX-51 inverted microscope with a 60x (NA 1.25) oil objective at room temperature and a Q-imaging Qicam (SN Q25830) camera. We used QCapturePro 6.0 as the acquisition software. To quantify LE/lysosome positioning, we assigned random file names and had a researcher blinded to the conditions score each cell. Cells with $>50 \%$ of the LAMP1 or RAB7 foci located next to and on one side of the nucleus were counted as displaying predominantly juxtanuclear LE/lysosomes. We scored approximately 100 cells for each condition in each experiment and repeated each experiment at least 3 times.

\section{Quantitative real-time RT-PCR and $X b p 1$ splicing assays}

We collected cells and isolated total RNA using Quick RNA Miniprep kits (Zymo Research). We synthesized cDNA with $700 \mathrm{ng}-2 \mu \mathrm{g}$ total RNA as a template, a T18 primer, and Moloney murine leukemia virus reverse transcriptase (New England Biolabs). Using a 
QuantStudio 3 real-time quantitative PCR machine (Life Technologies), we measured relative amounts of specific mRNAs with SYBR green as the fluorescent dye. All measurements were done in triplicate, and we quantified by comparing to serially diluted standard curve samples. Target mRNA primers were: Hgsnat, TCTCCGCTTTCTCCATTTTG and CGCATACACGTGGAAAGTCA, Blos1, CAAGGAGCTGCAGGAGAAGA and CCAGGAGGGtgaAgtAAGAGG, and Gfp, TCATCTGCACCACCGGCAAG and CAGCTCGATGCGGTTCACCA. We divided the abundance of each target mRNA by that for ribosomal protein 19 (Rpl19) mRNA from the same sample (primers for Rpl19: CTGATCAAGGATGGGCTGATand GCCGCTATGTACAGACACGA. For Xbpl splicing, we amplified cDNA with primers surrounding the regulated Xbpl splice site (AGAAGAGAACCACAAACTCCAG and GGGTCCAACTTGTCCAGAATGC) and ran the PCR products on a $2 \%$ agarose gel. We quantified the relative intensities of the spliced and unspliced Xbpl bands using ImageJ.

\section{Immunoblotting}

We trypsinized and collected cells, and lysed in RIPA buffer (25 mM Tris, $\mathrm{pH} 7.6,150$ $\mathrm{mM} \mathrm{NaCl}, 1 \% \mathrm{NP}-40,1 \%$ Na-deoxycholate, and $0.1 \%$ SDS) with protease and phosphatase inhibitors (Thermo Fisher Scientific). We then resolved soluble proteins using 12\% polyacrylamide NuPage Bis-Tris gels. We transferred the proteins to nitrocellulose and incubated for 1 hour in blocking buffer (5\% BSA, 0.05\% Tween20, 0.01\% Triton X-100, and TBS) at room temperature. We then probed using an anti-LC3B primary antibody (Sigma-Aldrich L7543, 1:1000, 4 C overnight), washed, and incubated with a secondary antibody (LiCor 926-32211, 1:10,000, 1 hour, RT). We used a LiCor Odyssey CLx Imager to scan the blot and quantified band intensities using the LiCor Image Studio software. We divided LC3B-II (processed, lipidated 
LC3B) band intensities by the sum of the intensities for unprocessed LC3B-I and processed LC3B-

II.

Flow cytometry and Annexin V/PI apoptosis assays

For measuring HTT-GFP abundance, we used a CytoFLEX 5 flow cytometer (Beckman Coulter) and the CytExpert version 2.3 software. For each sample in each experiment, we measured the median GFP intensity in a minimum of 4800 live cells and divided by the signal for untransfected cells.

To measure apoptosis, we used the Annexin V-Alexa Fluor 684 apoptosis assay kit (Invitrogen) according to the manufacturer's protocol, except that we used 5-fold less than the suggested annexin concentration to avoid excessive staining of control cells. We trypsinized and collected cells by centrifugation ( 800 xg, 5 min, RT), incubated in Annexin V (1:100, 15 min, 37 C) and Propidium Iodide (1 ug/mL, 2 min, 37 C), and measured fluorescence intensities by flow cytometry as above. 


\section{References}

1. Arrasate, M. \& Finkbeiner, S. Protein aggregates in Huntington's disease. Exp. Neurol. 238, 1 (2012).

2. Gan, L., Cookson, M. R., Petrucelli, L. \& La Spada, A. R. Converging pathways in neurodegeneration, from genetics to mechanisms. Nat. Neurosci. 21, 1300-1309 (2018).

3. Gutekunst, C.-A. et al. Nuclear and Neuropil Aggregates in Huntington's Disease: Relationship to Neuropathology. J. Neurosci. 19, 2522 (1999).

4. Kuemmerle, S. et al. Huntingtin Aggregates May Not Predict Neuronal Death in Huntington's Disease. (1999) doi:10.1002/1531-8249.

5. Tompkins, M. M. \& Hill, W. D. Contribution of somal Lewy bodies to neuronal death. Brain Res. 775, 24-29 (1997).

6. Ross, C. A. \& Poirier, M. A. What is the role of protein aggregation in neurodegeneration? Nat. Rev. Mol. Cell Biol. 2005611 6, 891-898 (2005).

7. Arrasate, M., Mitra, S., Schweitzer, E. S., Segal, M. R. \& Finkbeiner, S. Inclusion body formation reduces levels of mutant huntingtin and the risk of neuronal death. Nature 431, 805-810 (2004).

8. Saudou, F., Finkbeiner, S., Devys, D. \& Greenberg, M. E. Huntingtin Acts in the Nucleus to Induce Apoptosis but Death Does Not Correlate with the Formation of Intranuclear Inclusions. Cell 95, 55-66 (1998).

9. Iwata, A., Riley, B. E., Johnston, J. A. \& Kopito, R. R. HDAC6 and microtubules are required for autophagic degradation of aggregated Huntingtin. J. Biol. Chem. 280, 4028240292 (2005).

10. Olzmann, J. A., Li, L. \& Chin, L. S. Aggresome formation and neurodegenerative diseases: therapeutic implications. Curr. Med. Chem. 15, 47-60 (2008).

11. Kawaguchi, Y. et al. The Deacetylase HDAC6 Regulates Aggresome Formation and Cell Viability in Response to Misfolded Protein Stress. Cell 115, 727-738 (2003).

12. Erie, C. et al. Altered lysosomal positioning affects lysosomal functions in a cellular model of Huntington's disease. Eur. J. Neurosci. 42, 1941-1951 (2015).

13. Dodson, M. W., Zhang, T., Jiang, C., Chen, S. \& Guo, M. Roles of the Drosophila LRRK2 homolog in Rab7-dependent lysosomal positioning. Hum. Mol. Genet. 21, 135063 (2012).

14. Lie, P. P. Y. \& Nixon, R. A. Lysosome trafficking and signaling in health and neurodegenerative diseases. Neurobiol. Dis. 122, 94 (2019).

15. Bae, D., Moore, K. A., Mella, J. M., Hayashi, S. Y. \& Hollien, J. Degradation of Blos1 
mRNA by IRE1 repositions lysosomes and protects cells from stress. J. Cell Biol. 218, (2019).

16. Hollien, J. et al. Regulated Ire1-dependent decay of messenger RNAs in mammalian cells. J Cell Biol 186, 323-331 (2009).

17. Moore, K. \& Hollien, J. Ire1-mediated decay in mammalian cells relies on mRNA sequence, structure, and translational status. Mol Biol Cell 26, 2873-84 (2015).

18. $\mathrm{Pu}$, J. et al. BORC, a multisubunit complex that regulates lysosome positioning. Dev Cell 33, 176-188 (2015).

19. Hetz, C. \& Saxena, S. ER stress and the unfolded protein response in neurodegeneration. Nat. Rev. Neurol. 13, 477-491 (2017).

20. Shacham, T., Sharma, N. \& Lederkremer, G. Z. Protein Misfolding and ER Stress in Huntington's Disease. Front. Mol. Biosci. 6, 20 (2019).

21. Leitman, J., Ulrich Hartl, F. \& Lederkremer, G. Z. Soluble forms of polyQ-expanded huntingtin rather than large aggregates cause endoplasmic reticulum stress. Nat. Commun. 4, 2753 (2013).

22. Duennwald, M. L. \& Lindquist, S. Impaired ERAD and ER stress are early and specific events in polyglutamine toxicity. Genes Dev. 22, 3308-3319 (2008).

23. Henne, W. M., Buchkovich, N. J. \& Emr, S. D. The ESCRT Pathway. Dev. Cell 21, 77-91 (2011).

24. Sheehan, P., Zhu, M., Beskow, A., Vollmer, C. \& Waites, C. L. Activity-dependent degradation of synaptic vesicle proteins requires Rab35 and the ESCRT pathway. $J$. Neurosci. 36, 8668-8686 (2016).

25. Vaz-Silva, J. et al. Endolysosomal degradation of Tau and its role in glucocorticoid-driven hippocampal malfunction. EMBO J. 37, (2018).

26. Vietri, M., Radulovic, M. \& Stenmark, H. The many functions of ESCRTs. Nat. Rev. Mol. Cell Biol. 21, 25-42 (2020).

27. Schuck, S. Microautophagy - distinct molecular mechanisms handle cargoes of many sizes. J. Cell Sci. 133, (2020).

28. Sahu, R. et al. Microautophagy of Cytosolic Proteins by Late Endosomes. Dev. Cell 20, 131-139 (2011).

29. Jia, R., Guardia, C. M., Pu, J., Chen, Y. \& Bonifacino, J. S. BORC coordinates encounter and fusion of lysosomes with autophagosomes. Autophagy 13, 1-16 (2017).

30. Yordanov, T. E. et al. Biogenesis of lysosome-related organelles complex-1 (BORC) regulates late endosomal/lysosomal size through PIKfyve-dependent phosphatidylinositol3,5-bisphosphate. Traffic 20, 674-696 (2019).

31. Scott, I., Wang, L., Wu, K., Thapa, D. \& Sack, M. N. GCN5L1/BLOS1 Links Acetylation, Organelle Remodeling, and Metabolism. Trends Cell Biol. 28, 346-355 
(2018).

32. Zhang, C., Hao, C., Shui, G. \& Li, W. BLOS1 mediates kinesin switch during endosomal recycling of LDL receptor. Elife 9, 1-31 (2020).

33. Caballero, B. et al. Interplay of pathogenic forms of human tau with different autophagic pathways. Aging Cell 17, (2018).

34. Oshima, R. et al. ESCRT-0 dysfunction compromises autophagic degradation of protein aggregates and facilitates ER stress-mediated neurodegeneration via apoptotic and necroptotic pathways. Sci. Reports 201661 6, 1-15 (2016).

35. Kaul, Z. \& Chakrabarti, O. Endosomal sorting complexes required for ESCRTing cells toward death during neurogenesis, neurodevelopment and neurodegeneration. Traffic 19, 485-495 (2018).

36. AF, H. Extracellular Vesicles and Neurodegenerative Diseases. J. Neurosci. 39, 92699273 (2019).

37. Kalluri, R. \& LeBleu, V. S. The biology, function, and biomedical applications of exosomes. Science vol. 367 (2020).

38. Alexeyev, M. F., Fayzulin, R., Shokolenko, I. N. \& Pastukh, V. A retro-lentiviral system for doxycycline-inducible gene expression and gene knockdown in cells with limited proliferative capacity. Mol. Biol. Rep. 37, 1987-1991 (2010). 
Bae, Jones, Hollien

Figure 1

A $\quad$ B

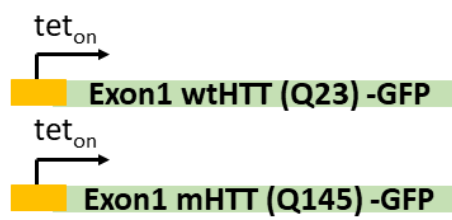

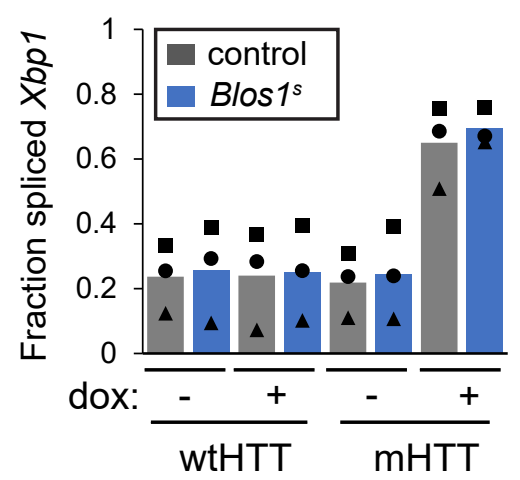

D
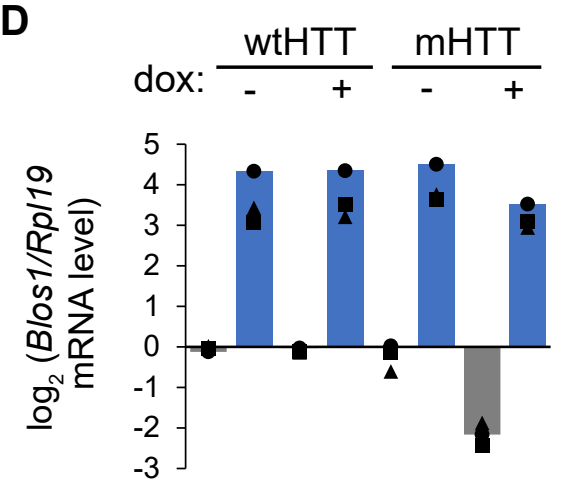

G

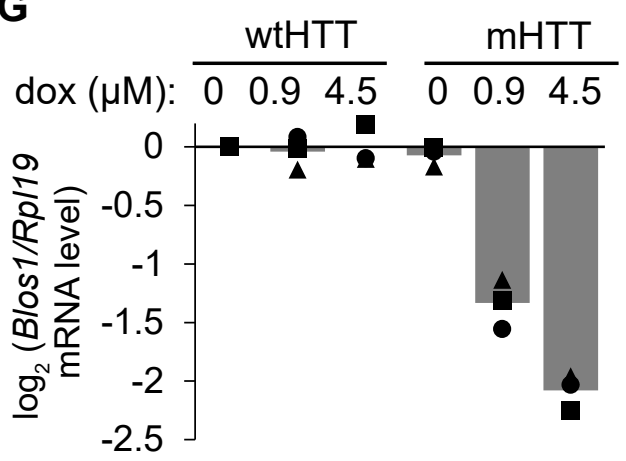

$\mathbf{E}$

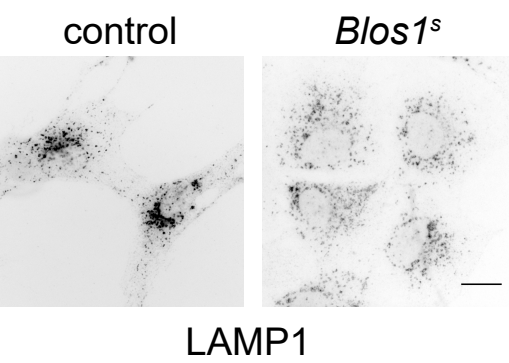

$\mathbf{H}$

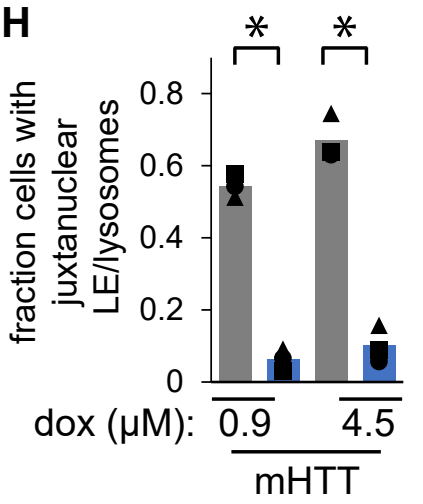

C dox: $\frac{\text { wtHTT }}{-+} \frac{\text { mHTT }}{-+}$
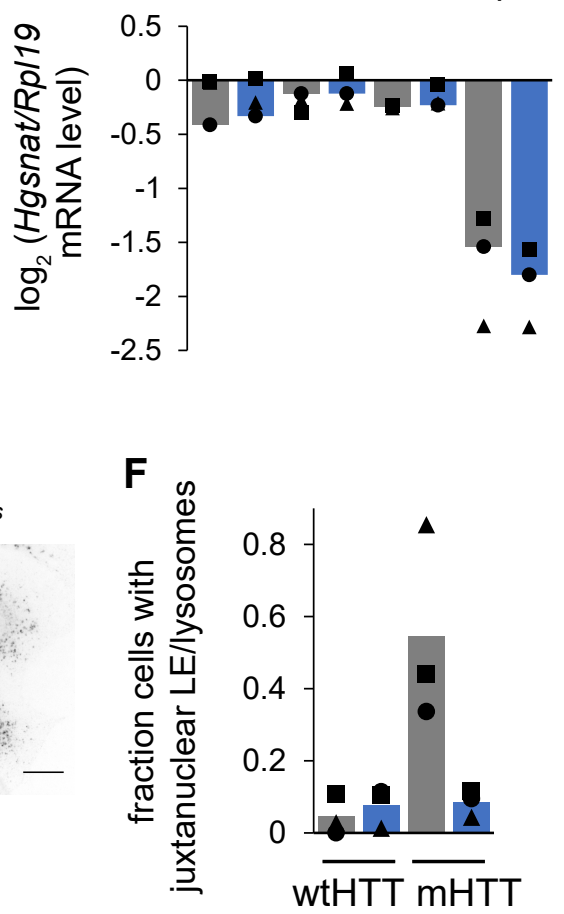
Bae, Jones, Hollien

Figure 2
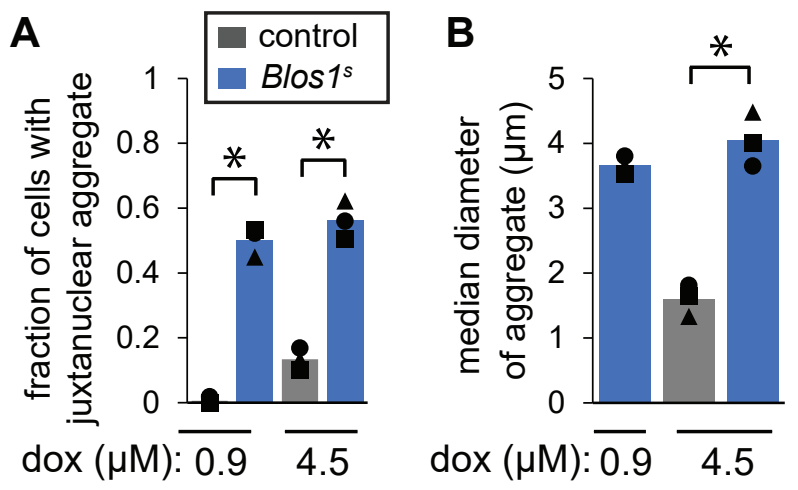

C
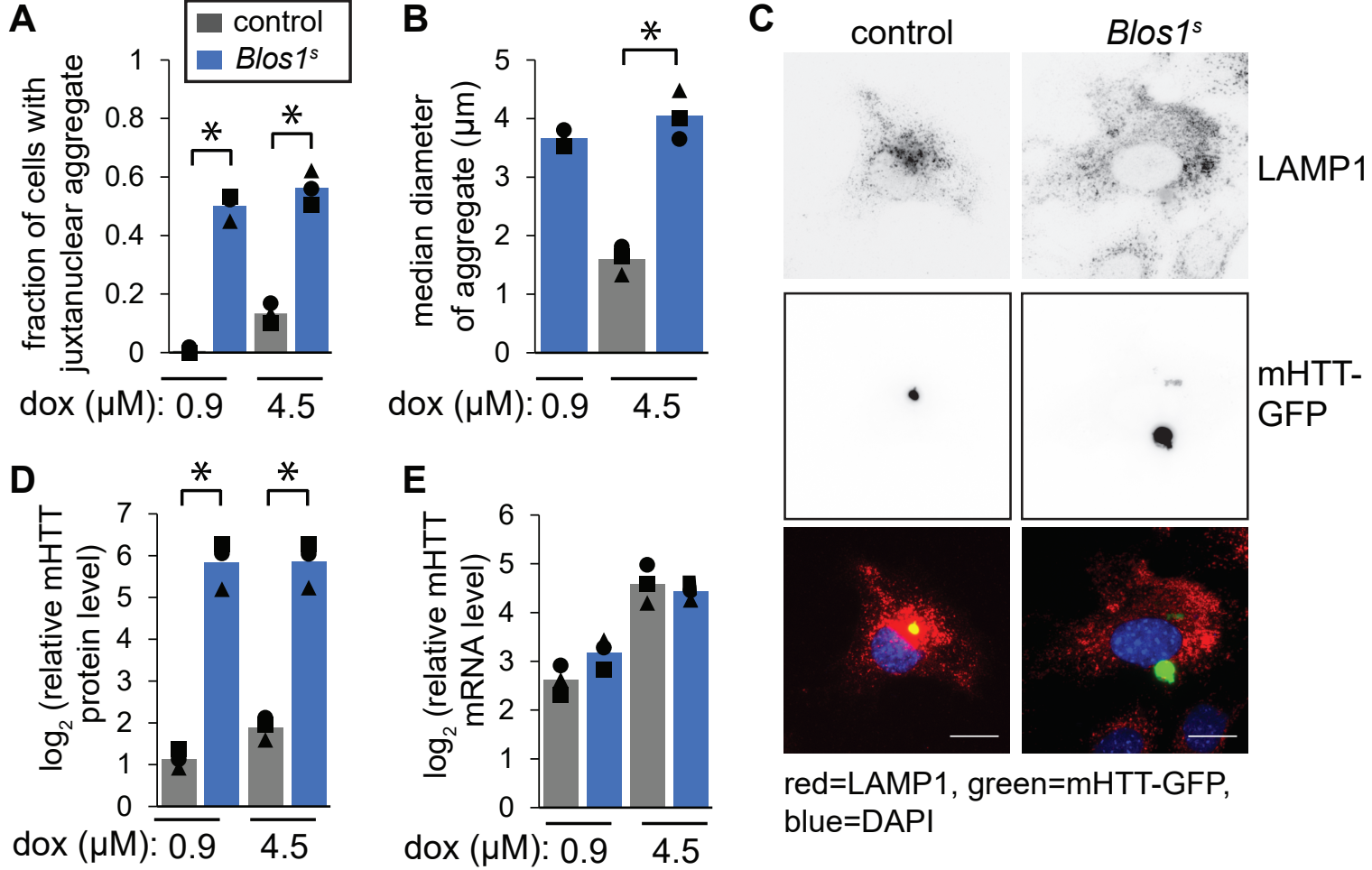

red=LAMP1, green=mHTT-GFP, blue=DAPI 
Bae, Jones, Hollien

\section{Figure 3}

A

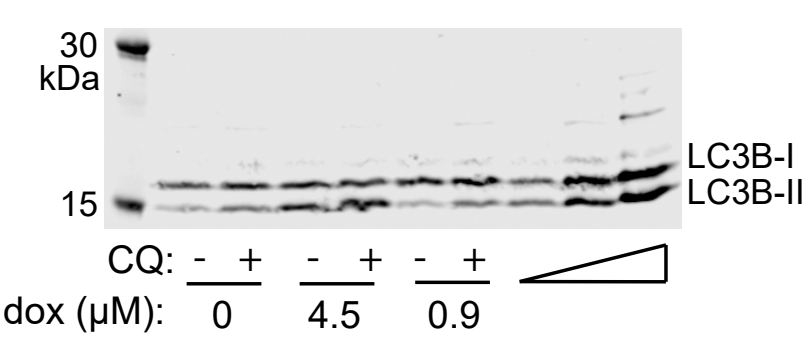

C

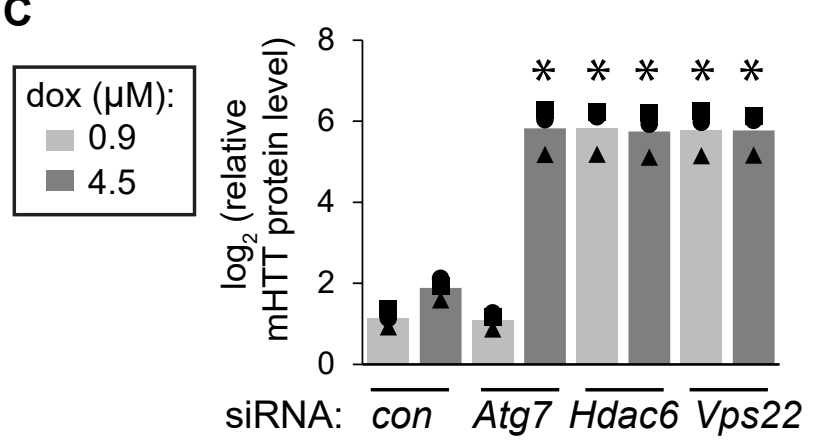

E

G

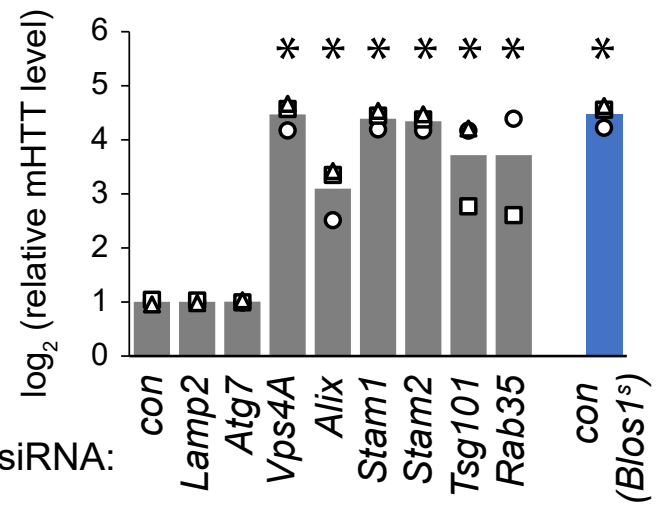

15

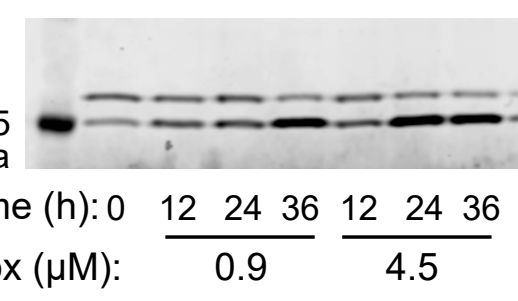

\section{.} $\operatorname{dox}(\mu \mathrm{M})$

H
B

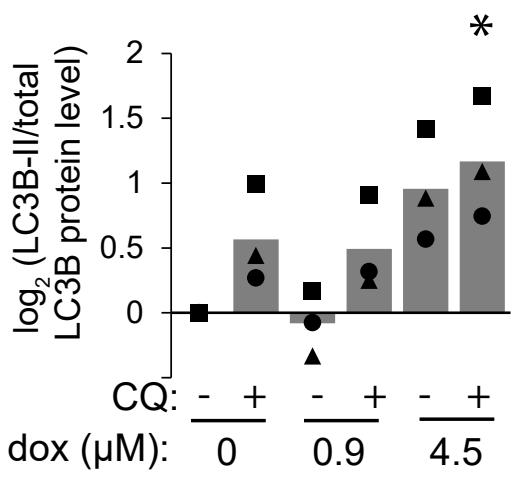

D

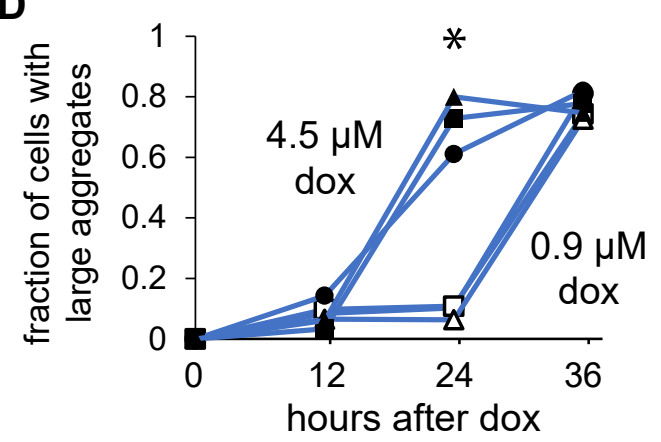

F

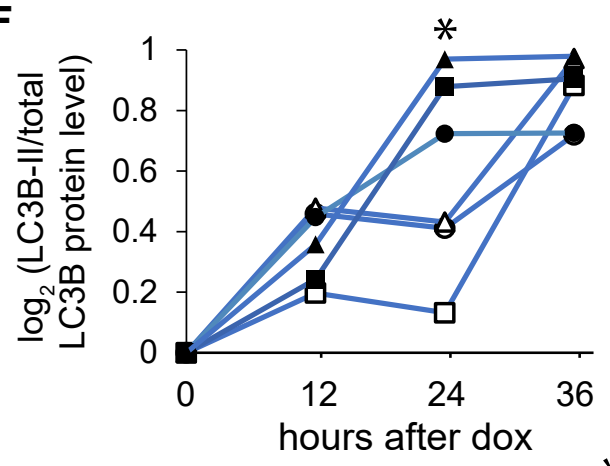

LC3B-I

LC3B-II

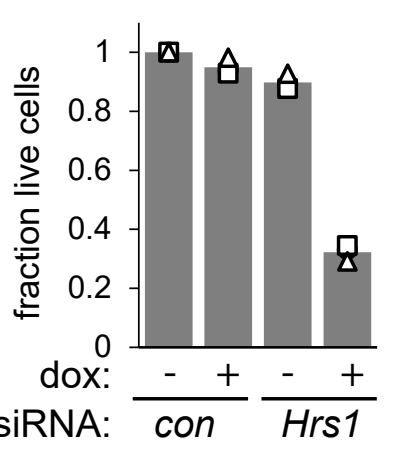

I
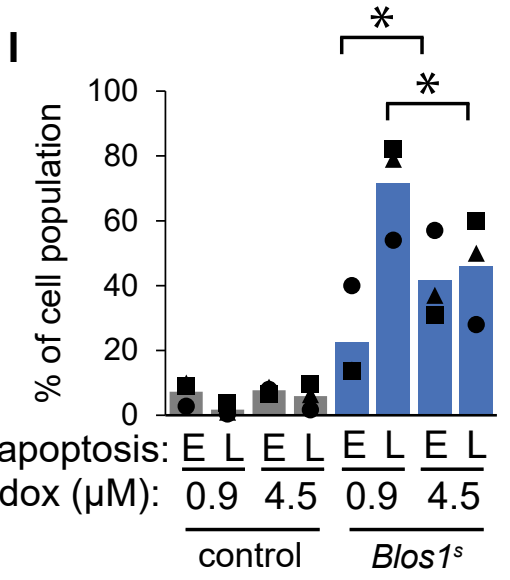


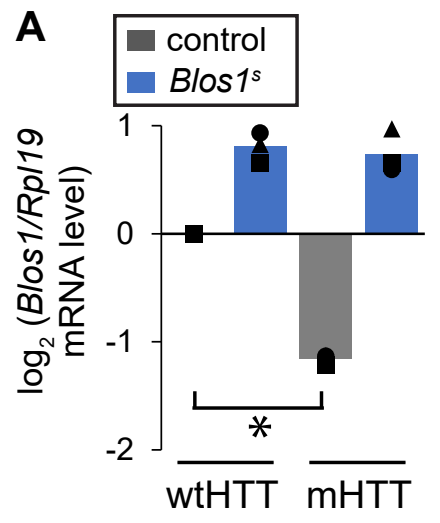

D

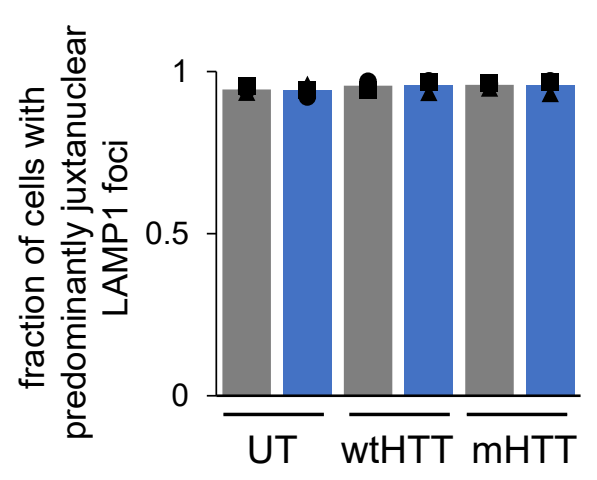

B

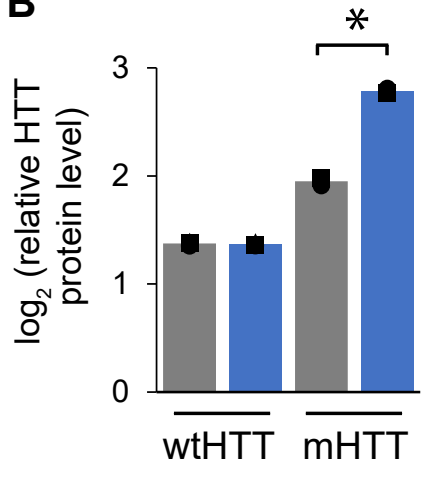

C

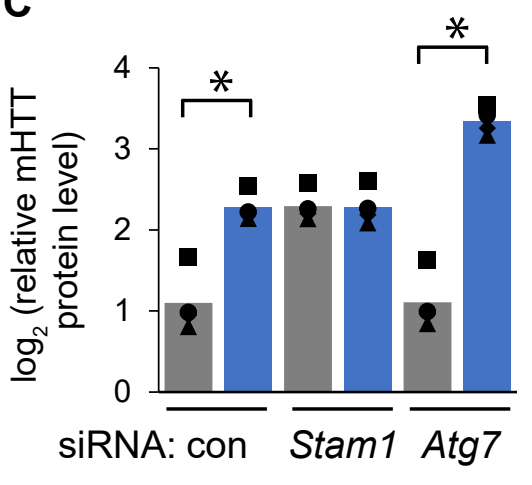

E
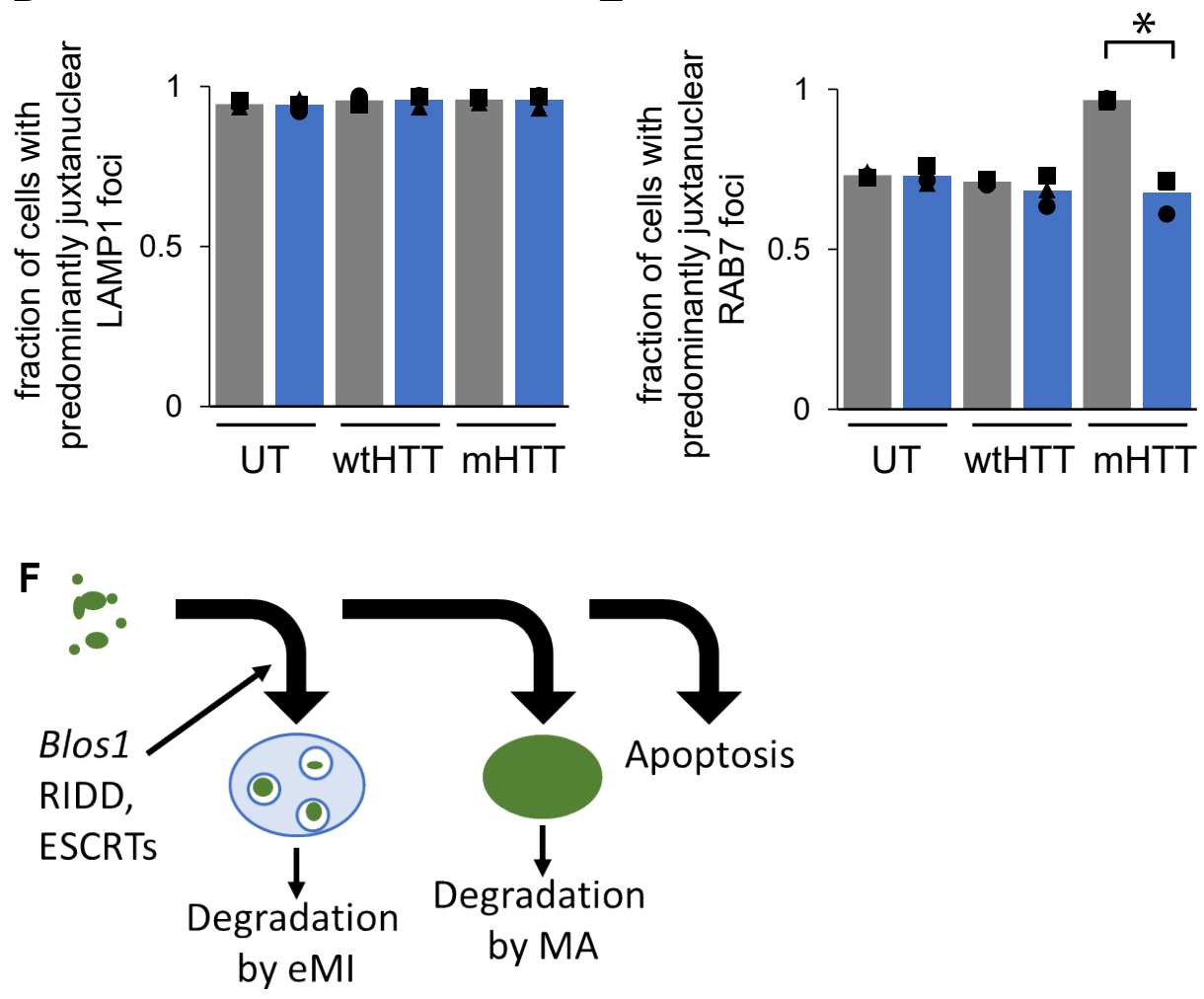